\title{
Ror $\mathrm{t}^{+}$innate lymphocytes and $\gamma \delta \mathrm{T}$ cells initiate psoriasiform plaque formation in mice
}

\author{
Stanislav Pantelyushin,, ${ }^{1}$ Stefan Haak, ${ }^{1}$ Barbara Ingold, ${ }^{2}$ Paulina Kulig, ${ }^{1}$ Frank L. Heppner, ${ }^{3}$ \\ Alexander A. Navarini, ${ }^{4}$ and Burkhard Becher ${ }^{1}$

\begin{abstract}
1Institute of Experimental Immunology, University of Zurich, Zurich, Switzerland. 2Institute of Pathology, Charité University Hospital, Berlin, Germany. ${ }^{3}$ Department of Neuropathology, Charité-Universitätsmedizin Berlin, Berlin, Germany. ${ }^{4}$ University Hospital Zurich,
\end{abstract} \\ Department of Dermatology, Zurich, Switzerland.
}

\begin{abstract}
Psoriasis is a common, relapsing inflammatory skin disease characterized by erythematous scaly plaques. Histological manifestations of psoriasis include keratinocyte dysregulation and hyperproliferation, elongated rete ridges, and inflammatory infiltrates consisting of $T$ cells, macrophages, dendritic cells, and neutrophils. Despite the availability of new effective drugs to treat psoriasis, the underlying mechanisms of pathogenesis are still poorly understood. Recent studies have shown that Aldara cream, used to treat benign skin abnormalities, triggers psoriasis-like disease in humans and mice and have implicated Th17 cells in disease initiation. Using this as a model, we found a predominant role for the Th17 signature cytokines IL-17A, IL-17F, and IL-22 in psoriasiform plaque formation in mice. Using gene-targeted mice, we observed that loss of Il17a, Il17f, or Il22 strongly reduced disease the severity of psoriasis. However, we found that Th17 cells were not the primary source of these pathogenic cytokines. Rather, IL-17A, IL-17F, and IL-22 were produced by a skin-invading population of $\gamma \delta$ T cells and ROR $\gamma \mathbf{t}^{+}$innate lymphocytes. Furthermore, our findings establish that ROR $\gamma \mathbf{t}^{+}$ innate lymphocytes and $\gamma \delta$ T cells are necessary and sufficient for psoriatic plaque formation in an experimental disease model that closely resembles human psoriatic plaque formation.
\end{abstract}

\section{Introduction}

Psoriasis is a complex, multifactorial disease, manifesting in an inflammatory response in the skin. The exact mechanistic underpinnings of psoriatic plaque formation are yet to be defined (1). It has been reported that psoriasis occurrences and relapses were inadvertently induced in patients that received topical treatment with Aldara, a cream containing imiquimod (IMQ) (2). In mice, the application of Aldara induces pathology that largely resembles the human disease phenotype of psoriasis (3). Moreover, it was shown to be similarly dependent on IL-23, and IL-17RA signaling, suggesting a role of Th17 effector cells (3-5). Recent studies have suggested a role for IL-17-producing $\gamma \delta \mathrm{T}$ cells in human psoriasis $(6,7)$. Here we report that initiation of plaque formation in the Aldara psoriasis model is dependent on ROR $\gamma \mathrm{t}^{+}$, skin-infiltrating $\gamma \delta \mathrm{T}$ cells, and innate lymphoid cells (ILCs). Our observations favor the notion that rather than Th cells, $V \gamma 4^{+} \gamma \delta \mathrm{T}$ cells and ILCs are the dominant and critical source of IL-17A, IL-17F, and IL-22 in the formation of acute psoriasiform lesions.

\section{Results and Discussion}

$I L-17 A, I L-17 F$, and $I L-22$ are critical for psoriatic plaque formation. After daily topical application of Aldara, we observed significant thickening, reddening, and scaling of the skin after 3 days (Supplemental Figure 1A; supplemental material available online with this article; doi:10.1172/JCI61862DS1). The clinical course of plaque formation and histopathology for the ear and the back skin were similar, with a slightly different kinetics (Supplemental Figure 1B). Aldara treatment resulted in hyperproliferation of keratinocytes

Authorship note: Stanislav Pantelyushin, Stefan Haak, and Barbara Ingold contributed equally to this work.

Conflict of interest: The authors have declared that no conflict of interest exists. Citation for this article: J Clin Invest. 2012;122(6):2252-2256. doi:10.1172/JCI61862. and disturbed epidermal differentiation, as indicated by acanthosis and hyperparakeratosis. An additional feature, reminiscent of Munro's microabscesses in human psoriasis, was the accumulation of terminal neutrophils (myeloperoxidase [MPO] staining) in the stratum corneum. Additionally, in the dermis extensive infiltration of leukocytes was observed (Supplemental Figure 1C).

Responsiveness to conventional antipsoriatic therapies is a hallmark for a valid psoriasis model (8). Ustekinumab (IL-12/23p40 monoclonal antibody) is one of the most efficacious treatments for psoriasis (4). Mice that were injected with a single dose of neutralizing murine IL-12/23p40 antibody displayed significantly less skin inflammation compared with control mice (Figure 1, A and B). Together with the recent finding that Aldara-induced psoriasiform plaques are responsive to UVB therapy (9), this disease model very closely resembles human disease visually and histologically and responds to known antipsoriatic therapies (1).

Skin-invading lymphocytes were analyzed for cytokine production at peak disease. IL-17F $\mathrm{F}^{+}$lymphocytes were substantially more abundant than IL-17A- and IL-22-producing cells in the skin of Aldara-treated mice (Figure 1C). We tested individual contributions of the implicated cytokines using $I l 17 \mathrm{a}^{-/-}, I l 17 \mathrm{f}^{-/-}$, and $I l 22^{-/-}$mice and found a significant reduction in the percentage change in skin thickness in all of the mutant mouse strains, compared with wild-type mice (Figure 1D). Histological analysis of the inflammation revealed a significant decrease in acanthosis in $I l 17 \mathrm{a}^{-/-}, I l 17 f^{-/-}, I l 22^{-/-}$mice compared with wildtype mice, with the most pronounced effects in mice lacking IL-17F and IL-22 (Figure 1E). The fact that there were more skin-invading cells secreting IL-17F than IL-17A is in line with the stronger disease resistance in $I l 17 f^{-/-}$compared with $1 l 17 \mathrm{a}^{-/-}$ mice. IL-17AF heterodimers have been described as sharing the biological properties with IL-17A and IL-17F (10), and we found significantly increased levels of IL-17AF heterodimers in the 


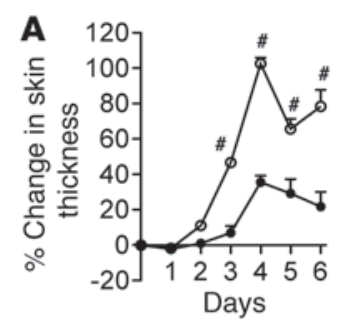

- Isotype Aldara $\rightarrow \alpha-I L-12 / 23 p 40$ Aldara
B
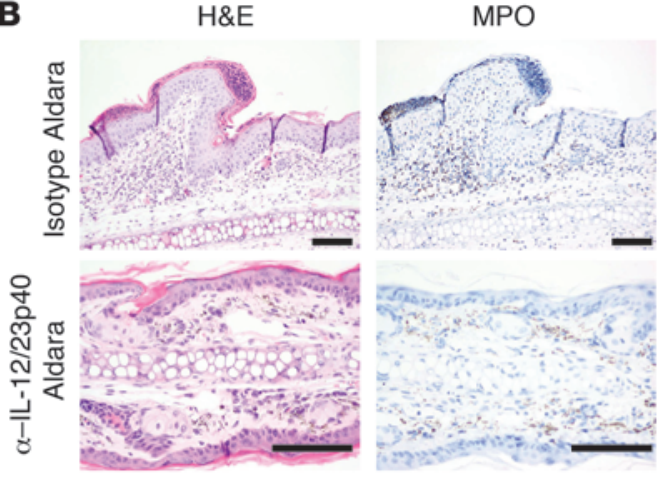

C
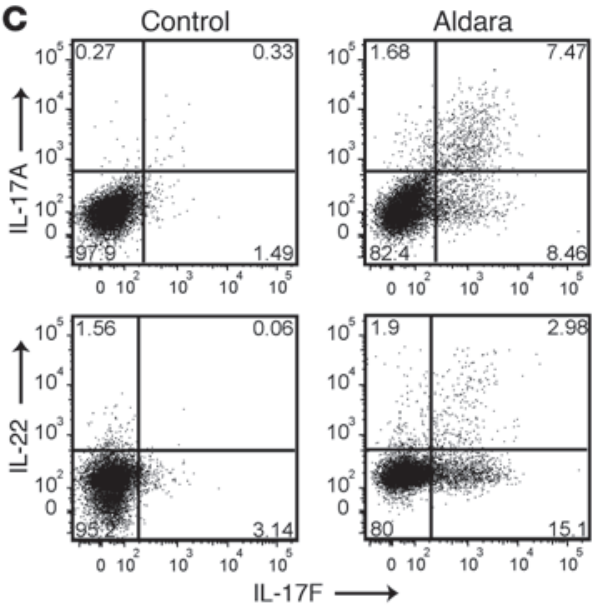

D

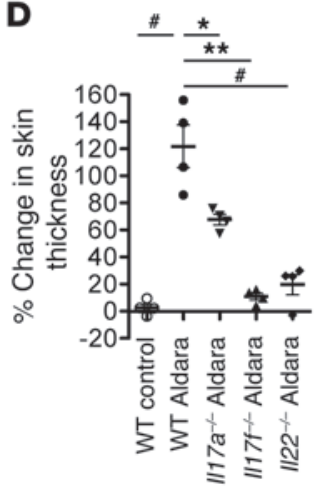

E

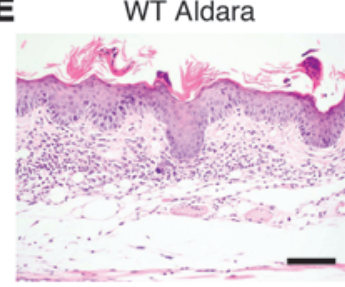

IL $17 f^{-/}$Aldara

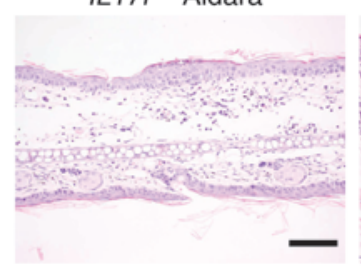

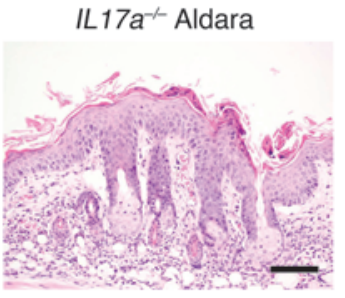

IL22 $2^{--}$Aldara

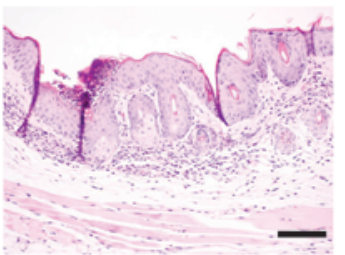

F
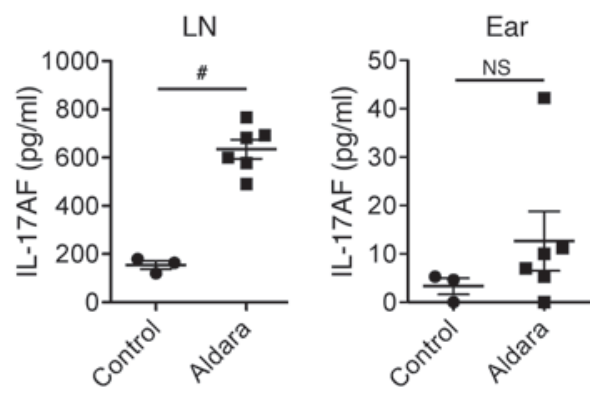

\section{Figure 1}

IL-17A, IL-17F, and IL-22 are important for psoriatic plaque formation. (A and B) WT mice were treated with Aldara and anti-IL-12/23p40 mAb or isotype control on day 2. (A) Kinetics of skin inflammation as percent increase in thickness over 6 days $(n=4)$. (B) Skin sections were stained with H\&E and anti-MPO. Scale bars: $100 \mu \mathrm{m}$. (C) Dot plots display the secretion of IL-17A, IL-17F, and IL-22 among CD45+ cells from the skin of wild-type mice on day 5 of Aldara treatment. (D and E) WT, $I / 17 a^{-1-}, I 17 f^{-1}$, and $I / 22^{-/-}$mice were treated with Aldara or control cream for 5 days. Scatter plot shows percent increase in skin thickness $(n=4)(D)$. Skin sections of Aldara-treated mice taken on day $6(E)$ were stained with H\&E. Scale bar: $100 \mu \mathrm{m}$. (F) IL-17AF heterodimer concentration was measured in the supernatant of LN or skin cells cultured for 24 hours using IL-17AF FlowCytomix Simplex kit (eBioscience). Each experiment was performed independently at least 3 times. ${ }^{\star} P<0.05$, ${ }^{\star \star} P<0.01$, ${ }^{\sharp} P<0.001$.

draining LNs, as well as slightly elevated levels in the inflamed skin of wild-type mice (Figure 1F).

An IL-17A-, IL-17F-, and IL-22-dependent mechanism of inflammation is in line with the TLR7 agonistic activity of IMQ (11), as skin inflammation could not be induced in $T \operatorname{lr} 7^{-/-}$mice. The induction of keratinocyte hyperproliferation in $\mathrm{Tlr}^{-/-}$mice, however, revealed TLR7-independent activities of Aldara (Supplemental Figure 1D). Apart from additional active ingredients in the Aldara cream formulation (M. van den Broek, unpublished observations), TLR7-independent pathways of keratinocyte activation have been previously reported by others (12).

$R O R \gamma t^{+} \gamma \delta T$ cells and innate lymphocytes are the main producers of IL-17A, IL-17F, and IL-22 in psoriasiform plaques. The transcription factor Roryt is required for IL-17 and IL-22 production $(13,14)$. To determine the main producers of these cytokines, we used RorcCre $\times$ EYFP fate-mapping mice. Surprisingly, only a small proportion of the cytokine producers were $\mathrm{CD} 4^{+} \mathrm{Th}$ cells. In contrast, about $80 \%$ of IL-17A as well as nearly $60 \%$ of IL-22 were produced by $\gamma \delta \mathrm{T}$ cells. The remaining IL-17A and IL-22 producers were ROR $\gamma \mathrm{t}^{+} \mathrm{CD} 3^{-}$ILCs, accounting for $10 \%$ of IL-17A and approximately $40 \%$ of IL-22 (Figure 2, A and B).

There is one report suggesting that human $\gamma \delta \mathrm{T}$ cells can potentially upregulate TLR7 expression and thus are equipped to respond to IMQ (15), but we failed to observe a direct effect of Aldara or IMQ in purified murine $\mathrm{V} \gamma 4 \mathrm{~T}$ cells (data not shown). There is, however, emerging evidence that conventional DCs initiate the immune response to IMQ (B. Clausen, unpublished observations).

At steady state, $\gamma \delta \mathrm{T}$ cells in the murine skin are primarily represented by ${\mathrm{V} \gamma 5^{+}}^{+}$resident epidermal cells (nomenclature according to ref. 16), termed dendritic epidermal T cells (DETCs), a cell population not found in human skin. Recently, it was shown that the dermis also contains ${\mathrm{V} \gamma 4^{+}}^{+}$resident cells expressing Roryt, CCR6, and IL-17A $(17,18)$. Staining for the $\gamma \delta$ lineage ( $\gamma$ chain) of the lymphocytes responsible for the observed Aldara skin inflammation revealed that there were two distinct populations (Supplemental Figure 2A). DETCs represented the TCR $\gamma \delta \delta^{\text {hi }}$ cells, and the major- 
A

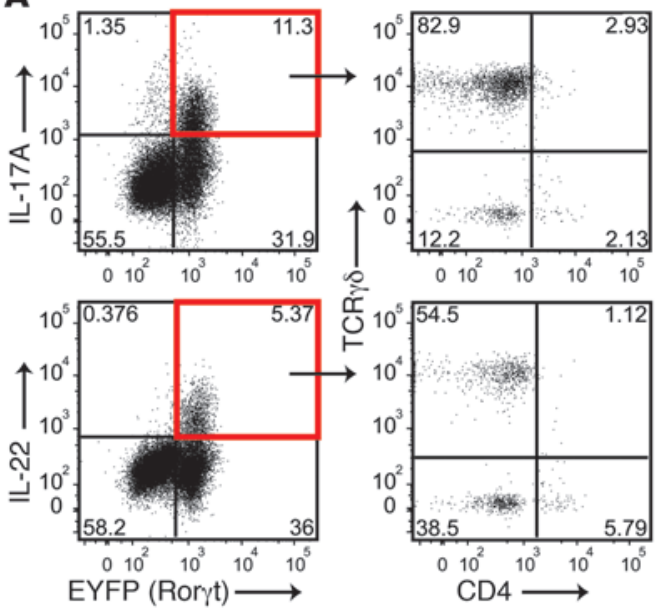

B

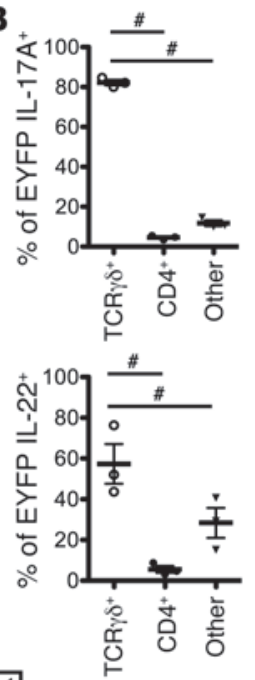

C
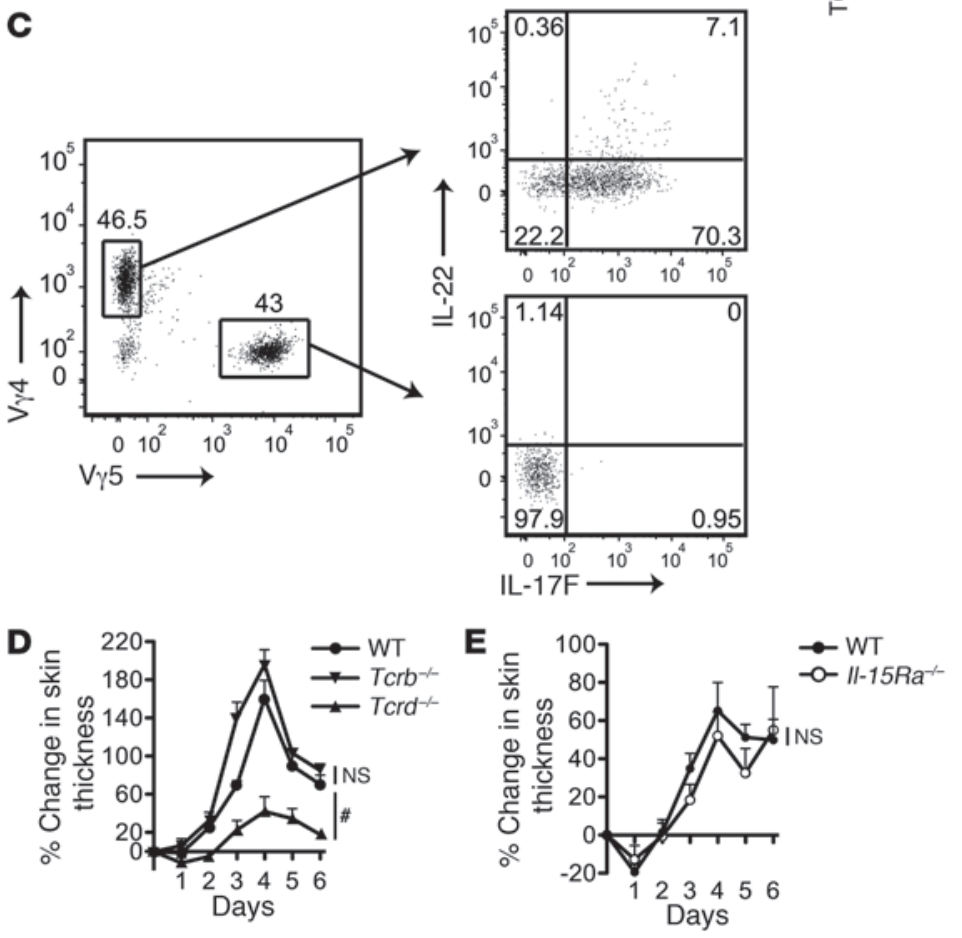

\section{Figure 2}

ROR $\gamma \mathrm{t}^{+} \gamma \delta \mathrm{T}$ cells and innate lymphocytes are the main producers of IL-17A, IL-17F, and IL-22 in psoriasiform plaques. (A) Intracellular cytokine staining in the skin of Rorc-Cre $\times$ EYFP mice after 5 days of Aldara treatment, gated on $\mathrm{CD} 45^{+}$cells $(n=3)$, with $(\mathrm{B})$ scatter plots showing percent distribution $(n=3)$. (C) Cytokine staining of $\mathrm{V} 4 \gamma^{+}$ versus $\mathrm{V} \gamma 5^{+}$for IL-17F and IL-22, pre-gated on TCR $\gamma \delta^{+}$ cells. (D and E) Kinetics of Aldara-induced skin inflammation in (D) WT versus $\mathrm{Tcrb}^{-/-}$and $\mathrm{Tcrd}^{-/-}$mice $(n=4)$ and (E) WT versus $/ / 15 \mathrm{ra}^{-1-}$ mice $(n=3)$, shown as the percent increase in skin thickness. Each experiment was performed independently at least 3 times. ${ }^{*} P<0.05,{ }^{\#} P<0.001$.

tion (6). Therefore these findings show that $\gamma \delta \mathrm{T}$ cells are indeed necessary for psoriasiform plaque formation.

$\mathrm{Tcrd}^{-/-}$mice lack both $\mathrm{V} \gamma 4^{+}$cells and DETCs. To formally rule out the involvement of DETCs in plaque formation, we treated wild-type and $\mathrm{Il}_{15 \mathrm{ra}^{-/-}}$mice, in which DETCs do not develop (Supplemental Figure $2 \mathrm{E}$ ), with Aldara and found inflammation to be indistinguishable (Figure 2E).

We found a similar increase in proliferation of $\mathrm{V} \gamma 4^{+}$cells in both the skin and skin-draining lymph nodes, suggesting that cytokine-secreting ${\mathrm{V} \gamma 4^{+}}^{+}$cells are not predominantly skin resident (Supplemental Figure 2F). A recent study on psoriasis in humans has demonstrated that a subset of $\gamma \delta$ T cells seems to play a role in disease development and that these cells express high levels of CCR6 and cutaneous leukocyte antigen (CLA) (7). Correspondingly, we found $\mathrm{V} \gamma^{+}{ }^{+}$cells to express the relevant skin-homing receptors CLA and CCR6, which could facilitate their recruitment to the skin (Supplemental Figure 2G).

$R O R \gamma t^{+}$innate lymphocytes are essential for psoriasiform plaque formation. Direct comparison of $\mathrm{Tcrd}^{-/-}$and $\mathrm{Rag}^{-1^{--}}$ mice revealed that both were relatively protected from Aldara-induced skin inflammation, with $\mathrm{Tcrd}^{-/-}$mice seemingly slightly more protected (Figure 3A). Cytofluorimetric comparison of the skin infiltrates revealed an increase in the number of ILCs in Rag1-/- compared with $\mathrm{Tcrd}^{-/-}$and wild-type mice. ILCs in $\mathrm{Rag}^{1^{-1}}$ mice had higher levels of CLA expression, but no major difference was found in regard to the expression of NKp46 or CD4 (Supplemental Figure 3, A-E).

ity of TCR $\gamma \delta^{\text {med }}$ cells were $V \gamma 4^{+}$(Supplemental Figure 2B). Upon disease induction, only the frequency (Supplemental Figure 2C) and total number of dermal $\mathrm{V} \gamma 4^{+}$cells increased, whereas DETC numbers were unaltered (Supplemental Figure 2D). Intracellular cytokine staining of $\gamma \delta$ subpopulations in the skin at peak disease revealed that $\mathrm{V} \gamma 4^{+}$cells produced IL-17F and IL-22, whereas DETCs did not (Figure $2 \mathrm{C}$ ), suggesting that $\mathrm{V} \gamma 4^{+}$cells drive the inflammatory skin alteration in this model.

To verify this, we compared the responses to Aldara in Tcrb ${ }^{-/-}$, $\mathrm{Tcrd}^{-/-}$, and wild-type mice. Tcrb ${ }^{-/-}$mice developed inflammation similar to that in wild-type mice. In contrast, $\mathrm{Tcrd}^{-/-}$mice had drastically lower, but still noticeable inflammation in the skin (Figure 2D). It is also possible that the $\gamma \delta \mathrm{T}$ cell pool in $\mathrm{Tcrb}^{-/-}$mice is altered due to the developmental changes in the $\gamma \delta$ lineage in the thymus (19). However, Cai and colleagues, using $\mathrm{Tcra}^{-/-}$mice, also found them to be fully susceptible to IL-23-induced plaque forma-
Analysis of Aldara-treated skin revealed a small population of Lin $^{-} \mathrm{NKp} 46^{-}$cells that were significant $(P=0.0076)$ producers of IL-22 (Figure 3B). Fate-mapping of ILCs in Rorc-cre $\times$ YFP $\times$ Rag $1^{-1-}$ mice showed that they invade the cellular niche of $\mathrm{T}$ cells and drive some degree of inflammation through increased production of IL-22 (Figure 3C). Using these mice, we also found ILCs to drastically increase IL-22 production in response to IL-23 (Supplemental Figure 3F). To confirm the role of ILCs in psoriasiform plaque formation, we analyzed the severity of inflammation by comparing Rag1 $1^{-/-}$mice, which lack T and NKT cells, with Rag2 $2^{-/-} \mathrm{Il} 2 \mathrm{rg}^{-/-}$, which additionally lack NK cells and ILCs. In con-

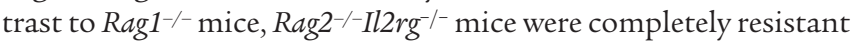
to Aldara-driven plaque formation (Figure 3D).

Thus far, our findings show the reliance of Aldara-induced psoriasis-like phenotype on ILCs and $\gamma \delta \mathrm{T}$ cells, which are largely dependent on the transcription factor ROR $\gamma$ t for their cytokine pro- 

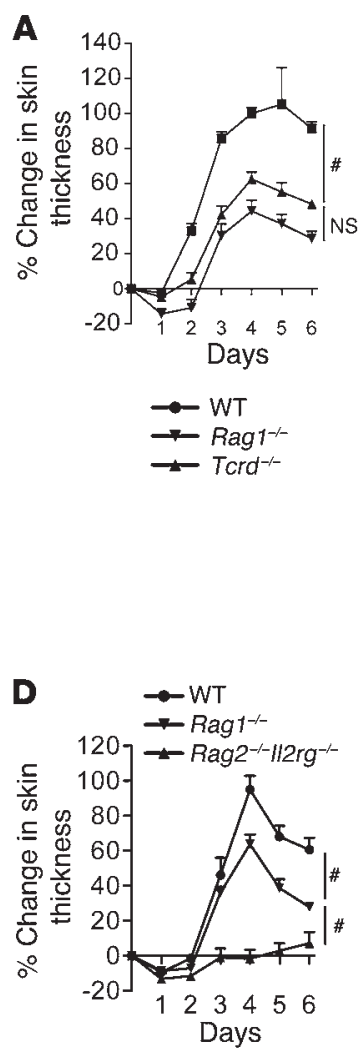
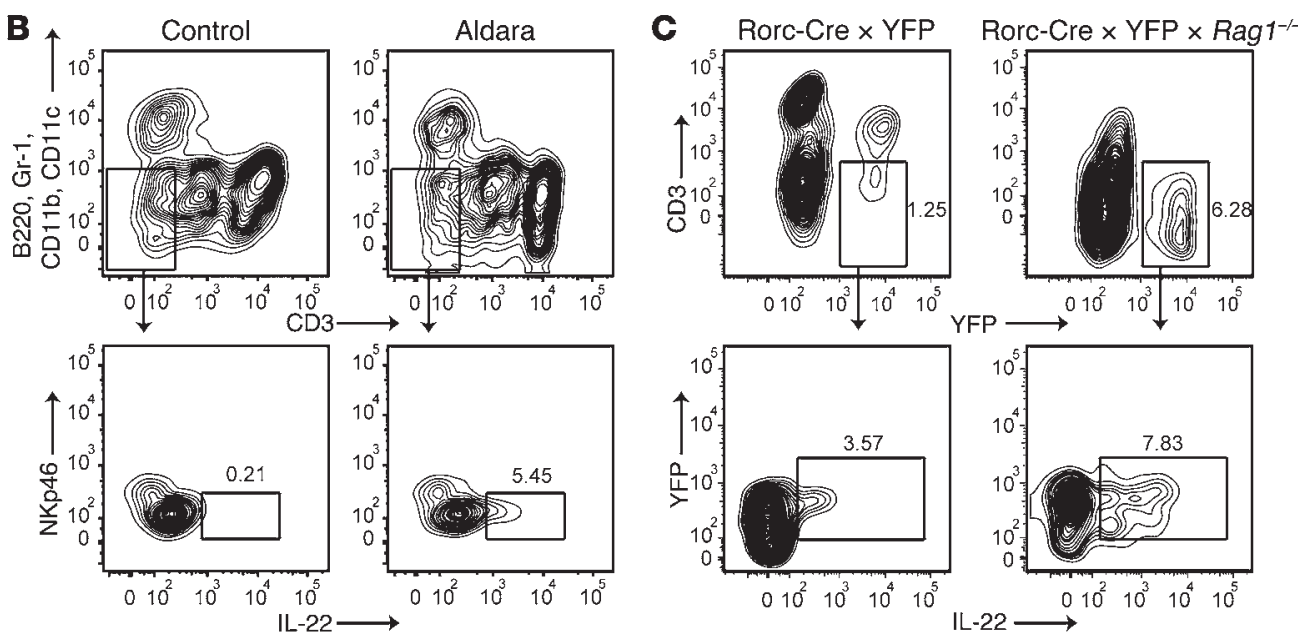
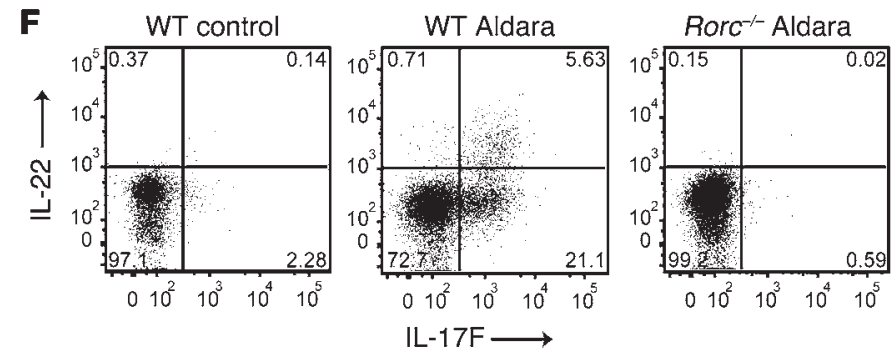

Figure 3

$\mathrm{ROR} \gamma \mathrm{t}^{+}$innate lymphocytes are essential for psoriasiform plaque formation. (A) Kinetics of Aldara-induced skin inflammation in WT versus Tcrd $^{-/-}$versus Rag 1-/- mice. (B) Cytokine staining in ILCs in the skin of Aldara- versus control-treated mice, pre-gated on Lin-CD45 ${ }^{+}$cells $(n=3)$. (C) Fate map analysis and IL-22 staining in the Aldara-treated skin of Rorc-Cre X EYFP and Rorc-Cre $\times$ EYFP $\times$ Rag 1-/- mice gated on CD45+

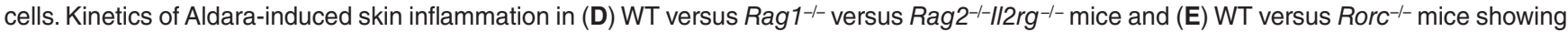
percent change in skin thickness $(n=3)$. (F) Staining for IL-17F and IL-22 in CD45+ cells from the skin of control and Aldara-treated WT versus Rorc $^{-/-}$mice $(n=3)$. Each experiment was performed independently at least 3 times. ${ }^{*} P<0.001$.

duction $(13,14)$. Consequently, as in $\mathrm{Rag}^{-/-} \mathrm{Il} 2 \mathrm{rg}^{-/-}$mice, no skin inflammation or thickening was observed in $\mathrm{Rorc}^{-/-}$mice (Figure $3 \mathrm{E})$. Moreover, IL-17A, IL-17F, and IL-22 were virtually absent in the skin of these mice compared with wild-type mice (Figure $3 \mathrm{~F}$ and data not shown). Therefore, Aldara-induced psoriasiform plaque formation seems to be completely dependent on the transcription factor ROR $\gamma t$ in $V \gamma 4^{+} \gamma \delta$ T cells and RAG-independent ILCs.

We set out to dissect the cellular underpinnings of psoriatic plaque formation and employed a recently described mouse model (3). Aldara-induced inflammatory lesions bear a substantial number of major hallmark histopathological and molecular features of human psoriatic plaques (1). The model is, however, limited to the simulation of psoriatic plaque formation, whereas the underlying natural pathogenic trigger of psoriasis, or any aspect of chronification of the inflammation, cannot be addressed. In a study parallel to ours, Cai and colleagues reported the pivotal role of dermal $\gamma \delta \mathrm{T}$ cells and their production of IL-17A in IL-23-induced skin inflammation in mice (6) and found evidence for their involvement in human psoriasis $(6,7)$. Some supporting evidence has also been reported in studies using the Aldara-induced psoriasis model $(6,20)$, which our study links to with an in-depth analysis of the pathophysiologic events during psoriatic plaque formation.

In summary, our data show that Ror $\gamma t$-dependent ILCs and $\gamma \delta$ $\mathrm{T}$ cells are necessary and sufficient to drive psoriasiform plaque formation in mice through the collective delivery of IL-17A, IL-17F, and IL-22 to the skin. The current notion that IL-23 induces Th17 cells stems from the observation that activated $\mathrm{T}$ cells are a major part of the skin-infiltrating immune cells and are a known source of these cytokines. Only recently have innate lymphocytes been acknowledged to be highly effective producers of these mediators. The fact that OKT3 antibody depleted human $\mathrm{T}$ cells was interpreted to indicate that psoriasis is dependent on $\mathrm{CD}^{+}$and $\mathrm{CD}^{+} \mathrm{T}$ cells (21), even though it also depletes $\gamma \delta$ $\mathrm{T}$ cells (22). Without dismissing adaptive immune processes in the etiology of psoriasis, our study does establish the sufficiency of a dysregulated innate immune compartment for psoriatic plaque formation. Thus, our proposed paradigm of lesion development not only provides a new basis for understanding the therapeutic efficacy of new biological drugs to treat human psoriasis, but may also lead to more in depth research on $\gamma \delta \mathrm{T}$ cell and ILC involvement in human disease.

\section{Methods}

Further information is available in Supplemental Methods.

Mice. C57BL/6 (wild-type) mice were obtained from Janvier. Rorc ${ }^{\text {GFP/GFP }}$ (in text referred to as $\mathrm{RorC}^{-/-}$), $\operatorname{Tcr} \beta \mathrm{\delta}^{-/-}, \mathrm{Rag}^{-/-}$, and $\mathrm{Rag}^{\mathrm{I}^{-/}} \mathrm{Il2 \textrm {rg } ^ { - / - }}$ mice were purchased from The Jackson Laboratory. Tcrb $\mathrm{cr}^{-/}$and $\mathrm{Tcrd} \mathrm{C}^{-/}$mice were bred from $\operatorname{Tcr} \beta \delta^{-/-}$mice. Rorc-Cre crossed with Rosa26-stop-eYFP mice (called 
Rorc-Cre $\times$ EYFP mice herein) were provided by A. Diefenbach (University of Freiburg, Freiburg, Germany), $I l 17 a^{-1-}$ mice by Y. Iwakura (University of Tokyo, Tokyo, Japan), Il17f/- mice by Merck Serono S.A., Il22-/- mice by J.C. Renauld (Université Catholique de Louvain, Yvoir, Belgium), and $\mathrm{Ill}_{15 \mathrm{ra}^{-1-}}$ mice by S. Bulfone-Paus (University of Giessen, Giessen, Germany).

Treatment. The mouse back was shaved, and a daily dose of $50 \mathrm{mg}$ of Aldara (5\% IMQ cream; 3M Pharmaceuticals) or control vehicle cream (Soft Kreme KA, Kantonsapotheke Zürich) was applied on the back and $5 \mathrm{mg}$ to each ear for 5-7 days. Anti-IL-12/23p40 treatment was performed by injection of the mice with $200 \mu \mathrm{g} / \mathrm{mouse}$ rat anti-mouse IL-12/23p40 mAb (C17.8) and respective IgG2A isotype control antibody (2A3, both Bio X Cell) on day 2 of Aldara application.

Cell preparation. Skin and ears were cut into small pieces and digested with $1 \mathrm{mg} / \mathrm{ml}$ collagenase type IA and $100 \mathrm{mg} / \mathrm{ml}$ DNase (Sigma-Aldrich) for 60 minutes at $37^{\circ} \mathrm{C}$. Isolation of leukocytes from the $\mathrm{LN}$ involved teasing the organs apart. Both were followed by filtering through $70-\mu \mathrm{m}$ cell strainers to obtain single-cell suspensions.

Antibodies. Cells were incubated with antibodies for 20 minutes at $4{ }^{\circ} \mathrm{C}$ For intracellular cytokine staining, cells were stimulated with PMA (Applichem) and ionomycin (Invitrogen) and treated with GolgiStop (BD) for 3 hours. After surface staining, cells were permeabilized according to the manufacturer's (BD) recommendations and stained intracellularly. Stainings were analyzed with a FACS LSRII Fortessa (BD). Post-acquisition analysis was performed with FlowJo (Tree Star) software.

Histology. Tissue samples were fixed in $4 \%$ paraformaldehyde and embedded in paraffin. Deparaffinized sections were stained using H\&E. Immunohistological staining for MPO (1:100, rabbit, Thermo Scientific) was performed by a Ventana Benchmark XT automated staining system according to the manufacturer's guidelines.

1. Nestle FO, Kaplan DH, Barker J. Psoriasis. N Engl J Med. 2009;361(5):496-509.

2. Gilliet M, et al. Psoriasis triggered by toll-like receptor 7 agonist imiquimod in the presence of dermal plasmacytoid dendritic cell precursors. Arch Dermatol. 2004;140(12):1490-1495.

3. van der Fits L, et al. Imiquimod-induced psoriasislike skin inflammation in mice is mediated via the IL-23/IL-17 axis. J Immunol. 2009;182(9):5836-5845.

4. Griffiths CE, et al. Comparison of ustekinumab and etanercept for moderate-to-severe psoriasis. NEngl J Med. 2010;362(2):118-128.

5. Hueber W, et al. Effects of AIN457, a fully human antibody to interleukin-17A, on psoriasis, rheumatoid arthritis, and uveitis. Sci Transl Med. 2010; 2(52):52ra72.

6. Cai Y, et al. Pivotal role of dermal IL-17-producing $\gamma \delta \mathrm{T}$ cells in skin inflammation. Immunity. 2011 35(1):1-15.

7. Laggner $U$, et al. Identification of a novel proinflammatory human skin-homing V $\gamma 9 \mathrm{~V} \delta 2 \mathrm{~T}$ cell subset with a potential role in psoriasis. J Immunol. 2011;187(5):2783-2793.

8.Nestle FO, Nickoloff BJ. Animal models of psoriasis: a brief update. J Eur Acad Dermatol Venereol. 2006;20(suppl 2):24-27.
Statistics. For disease severity, differences between groups were evaluated by 2-way ANOVA with Bonferroni's post hoc test. For analysis of scatter plots of maximum thickness comparing $\geq 3$ groups of mice, 1-way ANOVA with Bonferroni's post-test was used. Differences between two sets of data were evaluated by 2 -tailed Student's $t$ test. Data represent mean \pm SEM. $P \leq 0.05$ was considered statistically significant. All statistics were done using GraphPad Prism (GraphPad Software).

Study approval. All animal experiments were approved by the Swiss Cantonal Veterinary Office (33/2010; Zurich, Switzerland).

\section{Acknowledgments}

The work was funded by a project grant, (31003A-116070 to B. Becher) and a Sinergia grant (CRSII3_136203 to B. Becher) from the Swiss National Science Foundation, as well as by the Betty and David Koetser Foundation. We would like to thank S. Burkhard, F. Mair, V. Tosevski, D. Haefeli, J. Jaberg, and S. Hasler for technical assistance and M. Greter for manuscript preparation and discussion.

Received for publication November 8, 2011, and accepted in revised form March 14, 2012.

Address correspondence to: Burkhard Becher, Institute of Experimental Immunology, University of Zurich, Winterthurerstrasse 190 Y44-J92, 8057 Zürich, Switzerland. Phone: 41.44.635.3703; Fax: 41.44.635.6883; E-mail: becher@immunology.uzh.ch.

Stefan Haak's present address is: Columbia University Medical Center, Department of Microbiology and Immunology, New York, New York, USA.
9. Rácz E, et al. GATA3 Expression Is decreased in psoriasis and during epidermal regeneration; induction by narrow-band UVB and IL-4. PLoS One. 2011; 6(5):e19806.

10. Chang SH, Dong C. A novel heterodimeric cytokine consisting of IL-17 and IL-17F regulates inflammatory responses. Cell Res. 2007;17(5):435-440.

11. Hemmi H, et al. Small anti-viral compounds activate immune cells via the TLR7 MyD88-dependent signaling pathway. Nat Immunol. 2002;3(2):196-200.

12. Schön MP, Schön M, Klotz KN. The small antitumoral immune response modifier imiquimod interacts with adenosine receptor signaling in a TLR7- and TLR8-independent fashion. J Investig Dermatol. 2006;126(6):1338-1347.

13. Ivanov II, et al. The orphan nuclear receptor RORgammat directs the differentiation program of proinflammatory IL-17+ T helper cells. Cell. 2006; 126(6):1121-1133.

14. Sanos SL, et al. RORgammat and commensal microflora are required for the differentiation of mucosal interleukin 22-producing NKp46+ cells. Nat Immunol. 2009;10(1):83-91.

15. Gibbons DL, et al. Neonates harbour highly active gammadelta $T$ cells with selective impairments in preterm infants. Eur J Immunol. 2009;39(7):1794-1806.
16. Heilig JS, Tonegawa S. Diversity of murine gamma genes and expression in fetal and adult $\mathrm{T}$ lymphocytes. Nature. 1986;322(6082):836-840.

17. Gray EE, Suzuki K, Cyster JG. Cutting edge: Identification of a motile IL-17-producing gammadelta $T$ cell population in the dermis. J Immunol. 2011;186(11):6091-6095.

18. Sumaria $\mathrm{N}$, et al. Cutaneous immunosurveillance by self-renewing dermal gamma delta T cells. J Exp Med. 2011;208(3):505-518.

19. Silva-Santos B, Pennington DJ, Hayday AC. Lymphotoxin-mediated regulation of gammadelta cell differentiation by alphabeta $\mathrm{T}$ cell progenitors. $\mathrm{Sci}$ ence. 2005;307(5711):925-928.

20. Van Belle AB, et al. IL-22 is required for imiquimodinduced psoriasiform skin inflammation in mice. J Immunol. 2012;188(1):462-469.

21. Boyman O, Hefti HP, Conrad C, Nickoloff BJ, Suter $\mathrm{M}$, Nestle FO. Spontaneous development of psoriasis in a new animal model shows an essential role for resident $\mathrm{T}$ cells and tumor necrosis factoralpha. J Exp Med. 2004;199(5):731-736.

22. Keever-Taylor CA, et al. Complement-mediated T-cell depletion of bone marrow: comparison of T10B9.1A-31 and Muromonab-Orthoclone OKT3. Cytotherapy. 2001;3(6):467-481. 